\title{
Quench dynamics of correlated quantum dots
}

\author{
D.M. Kennes and V. Meden \\ Institut für Theorie der Statistischen Physik, RWTH Aachen University and \\ JARA-Fundamentals of Future Information Technology, 52056 Aachen, Germany
}

(Dated: December 22, 2018)

\begin{abstract}
We study the relaxation dynamics of a quantum dot with local Coulomb correlations coupled to two noninteracting leads which are held in grandcanonical equilibrium. Only charge degrees of freedom are considered and the dot is described by a model which in the scaling limit becomes equivalent to the interacting resonant level model. The time evolution of the current and dot occupancy resulting out of changes of the dot-lead coupling, the dots onsite energy, or the charging energy are studied. Abrupt and smooth parameter changes as well as setups with and without driving bias voltage are considered. For biased dots we investigate the often studied response after turning on the dot-lead coupling but also the experimentally more relevant case in which the voltage is turned on. We identify and explain a variety of interesting many-body effects and clarify the role of initial correlations.
\end{abstract}

PACS numbers: 05.10.Cc, 05.60.Gg, 72.10.Fk, 73.63.Kv

\section{INTRODUCTION}

In condensed matter systems at low temperatures twoparticle interactions lead to a variety of interesting quantum many-body effects which are not accessible by perturbative methods $\stackrel{1,2}{1}$ While in equilibrium one already has an elaborate understanding of many aspects of correlations in the field of nonequilibrium physics this stage is not reached yet. One can distinguish between the physics of the possible (nonequilibrium) steady state and that of the dynamics. Here we are interested in the time evolution resulting out of parameter changes in quantum dot systems with a few states tunnel coupled to two noninteracting leads. The dot electrons interact via the charging energy and the leads are held in grandcanonical equilibrium; possibly with two different chemical potentials, that is an applied bias voltage. The parameter changes might be abrupt or smooth on a scale set by the system's inherent time scales, with the ultimate limit of adiabatic changes; abrupt changes are often denoted as quenches. The progress in nanostructuring techniques allows to experimentally investigate quantum-dot setups with time dependent parameters in a rather controlled way $\underline{\underline{3}}$ They are of interest due to their potential for conventional as well as quantum information processing.

Several many-body methods successfully used in equilibrium were recently extended allowing to study the dynamics of correlated quantum dots out of nonequilibrium states; some of those being (mainly) analytical ${ }^{4-\underline{\underline{7}}}$ others purely numerical $. .8-12$ Only a few of these techniques have been used to treat Hamiltonians with explicitely time dependent parameters. We here apply a recently developed approximate semi-analytical method ${ }^{13}$ which is based on an extension of the functional renormalization group (RG) approach ${ }^{14}$ to Keldysh Green functions. ${ }^{15}-19$ It allows to study transport through quantum dots with a few energy levels, arbitrary time dependence of the single-particle parameters as well as the two-particle interaction $U$. The approximation of Ref. 13 is particu- larly suited for dots which only have charge degrees of freedom, but extensions towards spinful systems showing the Kondo effect are envisaged. In equilibrium and for the bias voltage driven nonequilibrium steady state functional RG based schemes which capture aspects of the (spin) Kondo effect have already been developed $20-22$ We here restrict ourselves to spinless systems and consider the interacting resonant level model (IRLM) $18,23-26$ and variants of it.

For small to intermediate $|U|$ our method was shown to provide reliable results for the dynamics of the IRLM on all time scales when applied to the standard setup of an initially uncorrelated and decoupled dot of arbitrary filling and arbitrary level position $\epsilon$, which at time $t=0$ is coupled to two grandcanonical leads held at different chemical potentials $\mu_{L}=V / 2$ and $\mu_{R}=-V / 2 ; U$ is turned on at $t=0$ as well. Here $V$ denotes the bias voltage. We use units with $e=\hbar=c=1$. The underlying initial state will be denoted as the standard initial state in the following and the quench protocol (turning on the level-lead coupling) as protocol (i). The time evolution of the dot occupancy and the current is characterized by an exponential decay towards the $U$-dependent steady state value with two rates, which for small $|U|$ are related by a factor of two. The decay is modulated by oscillations with frequencies given by $\left|\epsilon^{\mathrm{ren}} \pm V / 2\right|$ and power-law corrections,${ }^{26}$ where $\epsilon^{\text {ren }}$ denotes the (renormalized) level position. The functional $\mathrm{RG}^{13}$ in particular captures the crucial interaction induced renormalization of the decay rates ${ }^{18,23-26}$

Here we study additional protocols for the parameter changes leading to much richer dynamics. In protocol (ii) we start out in the standard initial state with $\epsilon=0$ and let the system evolve into its steady state which has dot filling $1 / 2$. We then quench the onsite energy to a large value. For finite bias voltage the resulting exponential decay of the occupancy towards its (new) steady-state value is repeatedly interrupted by plateau-like structures. They result from the interference of the oscillatory cur- 
rent and the oscillatory occupancies of the sites next to the central dot one. For $U=0$ the plateaus vanish. Protocol (iii) is related to (ii) with the crucial difference that the onsite energy is raised gradually over an interval $T_{\mathrm{q}}$. For $V=0$ and not too small $T_{\mathrm{q}}$ the occupancy smoothly decreases and oscillations are damped; the system adiabatically follows the parameter change. No characteristic correlation effects appear. This changes for $V>0$. For sufficiently large $T_{\mathrm{q}}$ we observe an initial increase of the dot occupancy when $\epsilon$ is gradually raised which vanishes for $U \rightarrow 0$. Studying the renormalization of the dot-lead couplings we obtain a detailed understanding of this surprising interaction effect. For both protocols we clarify the role of initial correlations 27 by turning on $U$ not at $t=0$ but at the time at which $\epsilon$ is raised. We find that in the present setup correlations build up rather quickly. In protocol (iv) we again start in the standard initial state with large $\epsilon$ as well as $\epsilon=0$ and $V=0$. After the system reached the steady state we abruptly turn on a bias voltage $V>0$. To allow for this experimentally more relevant situation (as compared to protocol (i)) we have to slightly extend the formalism presented in Ref. 13. Interestingly, for large $\epsilon$ the initial dynamics of the dot occupancy resulting out of this quench differs sizably from the one of protocol (i) with the bias being present at $t=0$. This already holds for $U=0$. For $\epsilon=0$ we identify interesting correlation effects. The protocols studied here exemplify the exceptional flexibility of our approach to the quench dynamics of correlated quantum dots. Others can be easily investigated as well.

Our paper is organized as follows. In Sect. [I] we introduce the quantum dot model and present the extension of the functional RG approach to allow for varying the bias voltage with time. Our results for the dynamics of the different quench protocols are presented in Sect. [II] We conclude with a brief summary in Sect. IV.

\section{MODEL AND METHOD}

\section{A. The quantum dot model}

Our model is given by the Hamiltonian

$$
H(t)=H^{\mathrm{dot}}(t)+\sum_{\alpha=L, R}\left[H_{\alpha}^{\text {lead }}+H_{\alpha}^{\text {coup }}(t)\right] .
$$

The dot part $H^{\text {dot }}(t)$ consists of three lattice sites with

$$
\begin{aligned}
& H_{0}^{\mathrm{dot}}(t)=\epsilon(t) n_{2}-U(t)\left(\frac{n_{1}}{2}+n_{2}+\frac{n_{3}}{2}\right) \\
&+\tau(t)\left(d_{1}^{\dagger} d_{2}+d_{2}^{\dagger} d_{3}+\text { H.c. }\right), \\
& H^{\mathrm{int}}(t)=U(t)\left(n_{1} n_{2}+n_{2} n_{3}\right),
\end{aligned}
$$

in standard second quantized notation. Here $n_{j}=d_{j}^{\dagger} d_{j}$ denotes the occupancy operator of the spinless fermionic level $j$. The levels (sites) are connected locally through a hopping matrix element $\tau>0$ and a Coulomb interaction
$U \geq 0$. Only the central site 2 can be moved in energy by changing the 'gate voltage' $\epsilon$. The second term in the single-particle part of the Hamiltonian is added for mere convenience so that $\epsilon=0$ corresponds to the point of particle-hole symmetry (half filling of the central site 2 in equilibrium). The two leads $\alpha=L, R$ are modeled as noninteracting,

$$
H_{\alpha}^{\text {lead }}=\sum_{k_{\alpha}} \epsilon_{k_{\alpha}} c_{k_{\alpha}}^{\dagger} c_{k_{\alpha}} .
$$

The left lead is tunnel-coupled to side 1 and the right one to site 3 through

$$
H_{\alpha}^{\text {coup }}(t)=t_{\alpha}(t) \sum_{k_{\alpha}} c_{k_{\alpha}}^{\dagger} d_{j_{\alpha}}+\text { H.c. },
$$

with $j_{L}=1$ and $j_{R}=3$. We explicitly allow for a time dependence of the parameters of $H^{\text {dot }}$ and $H_{\alpha}^{\text {coup }}$. We are here interested in the parameter regime in which band effects do not matter and consequently assume the leads to be in the wide band limit (for details see Ref. 13). The level-lead coupling is then parameterized by $\Gamma_{\alpha}\left(t^{\prime}, t\right)=\pi t_{\alpha}^{*}\left(t^{\prime}\right) t_{\alpha}(t) D_{\alpha}$, with the constant density of states $D_{\alpha}$ of lead $\alpha$. To reduce the number of parameters we focus on $\Gamma_{L}=\Gamma_{R}=\Gamma$. The leads are held at grandcanonical equilibrium with temperature $T=0$ and chemical potential $\mu_{L / R}= \pm V / 2$, with the bias voltage $V>0$.

In the scaling limit $\tau,|\epsilon|,|U|, V \ll \Gamma$ the model is equivalent to the field theoretical IRLM ${ }^{23}$ with a single energy level as the first and third dot sites can effectively be in-

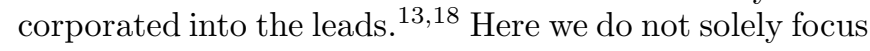
on the scaling limit but also consider setups with $\tau \lesssim \Gamma$ and $U \lesssim \Gamma$. For $\tau \approx \Gamma$ our model describes a dot with three levels. The linear geometry and the coupling of sites 1 and 3 to the leads implies a specific coupling of these three energy levels.

In Ref. 13 we gave a detailed account on how to obtain approximate expressions for the retarded, advanced and Keldysh Green functions and the corresponding selfenergies for Hamiltonians of the above type using functional RG. From the Green functions and self-energies the occupancies $\bar{n}_{j}$ of the dot sites and the left and right current $J_{\alpha}$ can be computed using standard formulas also given in Ref. 13. Consequently we here refrain from presenting any technical details on the functional RG and refer the interested reader to Ref. 13. Our approximation consists in keeping only the lowest order diagrams in the two-particle interaction $U$ on the right hand side of the $\mathrm{RG}$ flow equations for the vertex functions. Solving these differential equations however implies resummations of classes of diagrams leading to results beyond simple perturbation theory. We e.g. capture the renormalization of the decay rates characterized by a power-law behavior in the bare rates with a $U$-dependent exponent $\frac{18,23}{}$ as well as the power-law correction to the exponential decay in time of $\bar{n}_{2}(t)$ within protocol (i) with an interaction dependent exponent $\stackrel{13,26}{ }$ Both exponents come out correctly to leading order within our approximation 
and the method is thus reliable for $|U| \lesssim \Gamma$. We emphasize, that within the functional RG based scheme we can study the dynamics on arbitrary time scales. This has to be contrasted to numerical approaches to timedependent nonequilibrium transport through correlated quantum dots with which large times can currently not be reached. A detailed comparison of results for the standard protocol (i) to those obtained by other approaches can be found in Ref. 13. For completeness and comparison the physics of this protocol is briefly summarized in Sect. IIIA Within our approach the two-particle interaction leads to a (time dependent) renormalization of the single-particle parameters. To compute observables at the end of the RG flow one has to solve a single-particle problem with renormalized parameters indicated by the superscript 'ren'.

In protocol (iv) the bias voltage is turned on at a time $t_{\mathrm{q}}>0$. This type of time dependence is not directly captured by the formalism of Ref. 13 which we thus have to supplement. The necessary steps are described in the next subsection.

\section{B. Turning on the voltage}

In Ref. 13 we discussed how to handle time dependent couplings to the reservoirs via the time dependent hybridization $\Gamma_{\alpha}\left(t^{\prime}, t\right)$. We here show that a gauge argument can be used to shift time dependent chemical potentials $\mu_{\alpha}(t)$ into time dependent hybridizations; this way protocol (iv) is accessible to the approach of Ref. 13 as well.

For structureless wide band limit leads considered here raising the lead filling, and thus the chemical potential, is completely equivalent to raising all the energy levels of the leads. Therefore, we can replace the lead part of the Hamiltonian Eq. (4) by

$$
H_{\alpha}^{\text {lead }}=\sum_{k_{\alpha}}\left(\epsilon_{k_{\alpha}}+\mu_{\alpha}(t)\right) c_{k_{\alpha}}^{\dagger} c_{k_{\alpha}}
$$

and take the leads distribution functions as Fermi functions with zero chemical potential. We then apply the gauge transformation

$$
G(t)=e^{i \sum_{\alpha} \sum_{k_{\alpha}} \Omega_{\alpha}(t) c_{k_{\alpha}}^{\dagger} c_{k_{\alpha}}},
$$

where $\Omega_{\alpha}(t)$ is a suitable gauge field with $\Omega_{\alpha}(0)=0$. The transformed Hamiltonian reads

$$
\bar{H}(t)=G(t) H(t) G^{\dagger}(t)+i \dot{G}(t) G^{\dagger}(t),
$$

which shows that for setting $\dot{\Omega}_{\alpha}(t)=\mu_{\alpha}(t)$ the additional term in Eq. (6) originating from the time dependent chemical potential cancels with $i \dot{G}(t) G^{\dagger}(t)=$ $-\sum_{\alpha} \sum_{k_{\alpha}} \dot{\Omega}_{\alpha}(t) c_{k_{\alpha}}^{\dagger} c_{k_{\alpha}}$. Since $\left[H_{\alpha}^{\operatorname{coup}}(t), G(t)\right] \neq 0$ the hopping amplitude describing the level-lead coupling in $\bar{H}$ acquires an additional phase factor

$$
t_{\alpha}(t) \rightarrow t_{\alpha}(t) e^{i \Omega_{\alpha}(t)},
$$

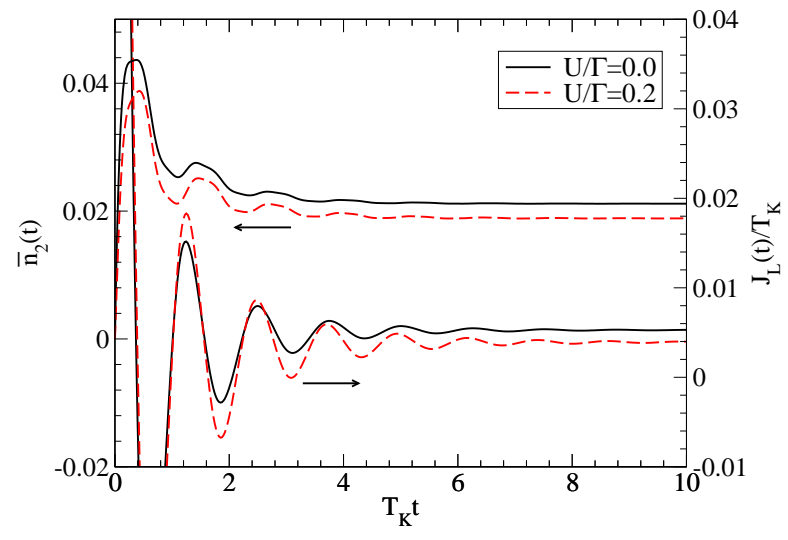

FIG. 1: (Color online) Time evolution of the occupancy $\bar{n}_{2}$ and the current leaving the left reservoir $J_{L}$ for the IRLM in protocol (i). The parameters are $\tau / \Gamma=0.0025, \epsilon / T_{\mathrm{K}}=10$, and $V / T_{\mathrm{K}}=10$. The universal energy scale $T_{\mathrm{K}}$ is renormalized by the local Coulomb interaction $U$ (see the text).

where $\Omega_{\alpha}(t)=\int_{0}^{t} d t^{\prime} \mu_{\alpha}\left(t^{\prime}\right)$. The transformed Hamiltonian can then be treated as outlined in Ref. 13 .

\section{RESULTS FOR THE QUENCH DYNAMICS}

In this section we present and discuss our functional RG results for the time evolution of the occupancies $\bar{n}_{j}$ and the currents $J_{\alpha}$ induced by the quench protocols defined in the introduction. The focus is on the correlation effects. For comparison and to gain a deeper understanding we present $U=0$ results when necessary. Already now we emphasize that some of the interaction effects cannot directly be linked to the renormalization of the decay rate, which in the standard setup protocol (i) was found to be the most dominant effect of the two-particle interaction. ${ }^{18,24-26}$ To keep our presentation self-contained the physics of protocol (i) is briefly summarized in the following.

\section{A. Protocol (i)}

In protocol (i) we start out with an initially empty dot decoupled from the two grandcanonical leads held at chemical potentials $\mu_{L / R}= \pm V / 2$. The coupling to the reservoirs is turned on at time 0 . Figure 1 shows the time evolution of the level occupancy $\bar{n}_{2}$ and the current $J_{L}$ leaving the left lead in the scaling limit (the IRLM) for $\tau / \Gamma=0.0025, \epsilon / T_{\mathrm{K}}=10$, and $V / T_{\mathrm{K}}=10$. Here $T_{\mathrm{K}}$ is the universal energy scale defined by the equilibrium charge susceptibility $\underline{18,23,25,26}$

$$
\chi=\left.\frac{d \bar{n}_{2}}{d \epsilon}\right|_{\epsilon=0}=-\frac{2}{\pi T_{\mathrm{K}}} .
$$

For $U=0$ it is given by $4 \tau^{2} / \Gamma$, but gets strongly renormalized by the interaction. ${ }^{18,23}-26$ It increases for small 
to intermediate repulsive interactions. The exponential decay of $\bar{n}_{2}$ and $J_{\alpha}$ towards their respective $U$-dependent steady-state values is characterized by two relaxation rates. For small $U$ they are approximately given by $T_{\mathrm{K}}$ and $T_{\mathrm{K}} / 2$. The decay with the smaller rate is accompanied by a power-law correction in $t$ with a $U$-dependent exponent $\underline{13,18,26}$ Since this is only a small correction to the exponential decay the main effect of the interaction is the strong enhancement of the relaxation rates for $U>0$. For $\left|\epsilon^{\mathrm{ren}} \pm V / 2\right| \gg T_{\mathrm{K}}, 1 / t$ the decay of the occupancy $\bar{n}_{2}(t)$ is overlayed by oscillations with the two frequencies $\left|\epsilon^{\mathrm{ren}} \pm V / 2\right|$, where the renormalization of the onsite energy $\epsilon \rightarrow \epsilon^{\text {ren }}$ is of order $(U / \Gamma)^{2}$ and thus small. In the current leaving the left (right) lead the frequency $\left|\epsilon^{\mathrm{ren}}-V / 2\right|\left(\left|\epsilon^{\mathrm{ren}}+V / 2\right|\right)$ dominates $\frac{13,18,26}{10}$ Comparing the curves for $U=0$ and $U>0$ in Fig. 1 it is apparent that the two-particle interaction leads to an increase of the oscillation amplitudes.

\section{B. Protocol (ii)}

We next study the response of a system, which after being prepared according to protocol (i) has reached a steady state, to the sudden increase of $\epsilon$ from $\epsilon_{\text {ini }} / \Gamma=0$ to the rather large value $\epsilon_{\text {final }} / \Gamma=2$ at time $t_{\mathrm{q}}>0$. We choose $\tau / \Gamma=0.5$, which is no longer deep in the scaling limit. The time-evolution for $t<t_{\mathrm{q}}$ (see Fig. (2) still shows the main features found for the IRLM discussed in the last subsection.

Figure 2 shows the time evolution of the occupancies of the three sites as well as $J_{L}$ for $V / \Gamma=2$. The occupancy of the middle site and the current drop quickly after the $\epsilon$ quench at $\Gamma t_{\mathrm{q}}=20$ as the onsite energy is raised far above the chemical potential. Whereas in the noninteracting case $\bar{n}_{2}(t)$ smoothly decreases (up to weakly developed shoulders; dashed line in the upper panel) this depletion is periodically interrupted for $U>0$ (solid line; see the ellipses in the upper panel). This effect, which clearly goes beyond the renormalization of the decay rates, can be understood as follows. The interaction enhances the coherence of the system leading to an increase of the oscillation amplitudes as discussed for protocol (i). Plateaulike features show up for time regimes where maxima of the current leaving the left reservoir coincide with the decreasing part of the oscillatory occupancy of the first site $\bar{n}_{1}(t)$. The combination of these tendencies temporarily stops the depletion process on the central site leading to the plateaus. These time regimes are marked with $\mathrm{A}$ in Fig. 2, For times in which minima in the left lead's current coincide with an increase of $\bar{n}_{1}$, marked with $\mathrm{B}$ in Fig. 2 the depletion of the central site is even enhanced compared to the noninteracting case. The comparison to the curve for $U / \Gamma=1$ but $V=0$ (dashed-dotted line in the upper panel) shows that a finite bias voltage is vital for the development of the plateaus.

To understand the periodic interception of the decay on a more quantitative level we determine the frequencies

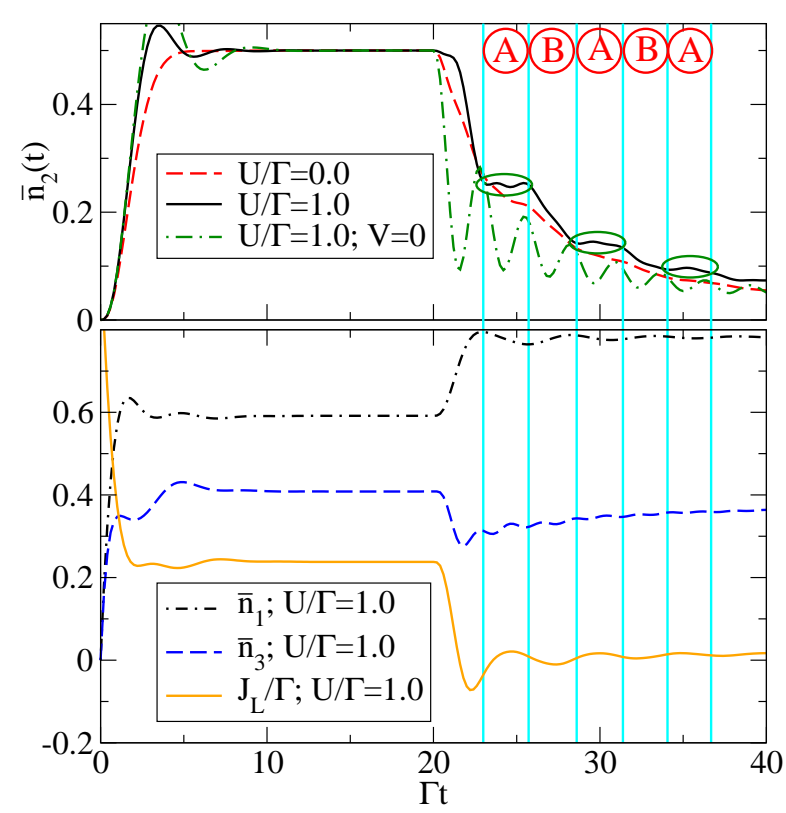

FIG. 2: (Color online) Top: Time evolution of the occupancy $\bar{n}_{2}$ in protocol (ii). The quench from $\epsilon_{\text {ini }} / \Gamma=0$ to $\epsilon_{\text {final }} / \Gamma=2$ is performed at time $\Gamma t_{\mathrm{q}}=20$. At this time the system has reached a steady state after initially being prepared according to protocol (i). The other parameters are $\tau / \Gamma=0.5$ and $V / \Gamma=2$. The plateau-like features interrupting the exponential decay beyond $\Gamma t_{\mathrm{q}}=20$ are highlighted by ellipses. For comparison $\bar{n}_{2}(t)$ for $U / \Gamma=1$ and $V=0$ is shown as well. Bottom: Time evolution of $\bar{n}_{1 / 3}$ and $J_{L}$.

expected for $t>t_{\mathrm{q}}$ from protocol (i). For the noninteracting case those are given by Eq. (A.5) of Ref. 13 considering the poles associated to the smallest relaxation rates (which dominate at large times). As a first approximation one can use the same formula with the bare parameters replaced by the renormalized ones taken for $t \rightarrow \infty$. This gives an estimate of the corresponding frequencies in the interacting case. For the parameters of Fig. 2 we obtain

$$
\begin{aligned}
& \omega^{\text {ren }}+V / 2 \approx 3.23747 \Gamma \\
& \omega^{\text {ren }}-V / 2 \approx 1.23747 \Gamma .
\end{aligned}
$$

These two frequencies correspond roughly to the frequencies associated to the periodic appearance of the plateaus (smaller frequency) as well as the oscillation frequencies within the plateaus (higher frequency).

\section{Protocol (iii)}

We now focus on the case in which the change of the onsite energy described in the last subsection is not abrupt, but is performed with constant velocity over the time interval $T_{\mathrm{q}}$ centered around $t_{\mathrm{q}}$ from $\epsilon_{\text {ini }} / \Gamma=0$ to $\epsilon_{\text {finial }} / \Gamma=2$. To ensure that the system has fully reached its steady state at time $t=t_{\mathrm{q}}-T_{\mathrm{q}} / 2$ we modify the initial 


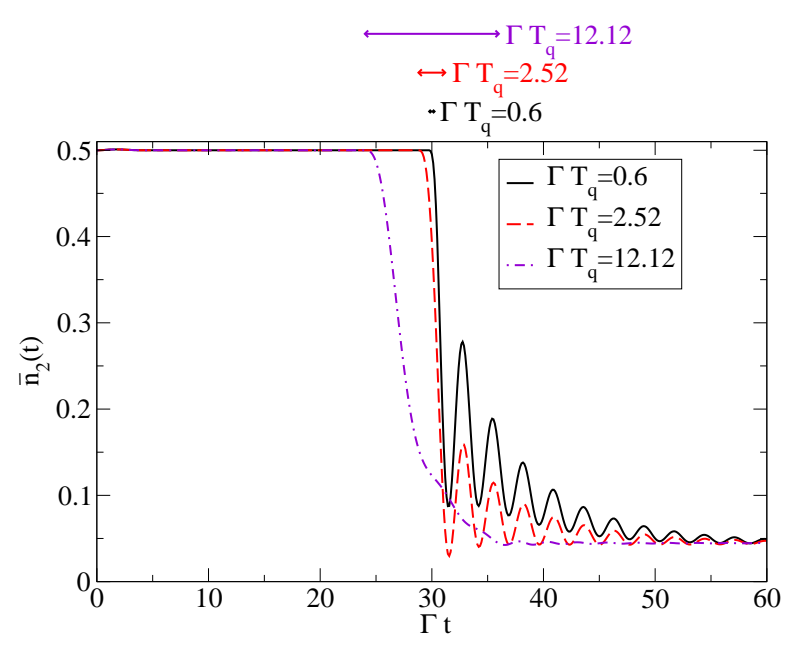

FIG. 3: (Color online) Time evolution of $\bar{n}_{2}$ for a change of $\epsilon$ performed with constant velocity in the time interval $T_{\mathrm{q}}$ (protocol (iii)). The parameter are chosen as in Fig. 2 but with $V=0$. Arrows above the graph indicate the time interval over which the change of $\epsilon$ is performed.

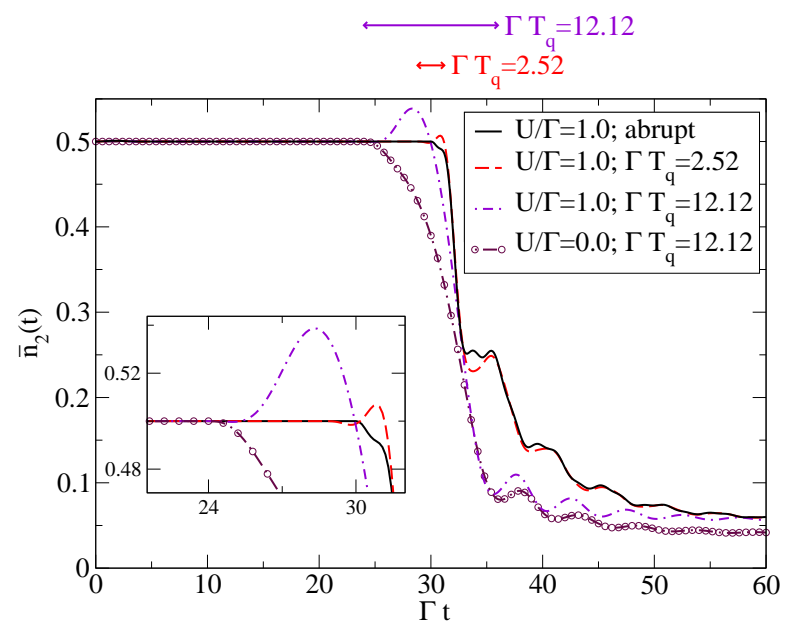

FIG. 4: (Color online) The same as in Fig. 3but with $V / \Gamma=2$. For comparison the time evolution after an abrupt quench (see Fig. 21) is shown as well. The inset shows a zoom-in of the region in which $\bar{n}_{2}$ (initially) increases. Arrows above the graph indicate the time interval over which the change of $\epsilon$ is performed.

preparation according to protocol (i) by starting at $t=0$ with an initial density matrix with occupancy $1 / 2$ on its diagonal.

We start the discussion with the unbiased case $V=0$ and choose the other parameters as $\tau / \Gamma=0.5$ and $U / \Gamma=$ 1. Figure 3 shows the time evolution of $\bar{n}_{2}$ for different $T_{\mathrm{q}}$ (see the arrows above the graph) with $\Gamma t_{\mathrm{q}}=30$ fixed. As expected the amplitude of the oscillations decreases with increasing $T_{\mathrm{q}}$. In the limit $T_{\mathrm{q}} \rightarrow \infty$ the system adiabatically follows the parameter change and the oscillations disappear.

Next we consider the biased case with $V / \Gamma=2$ shown

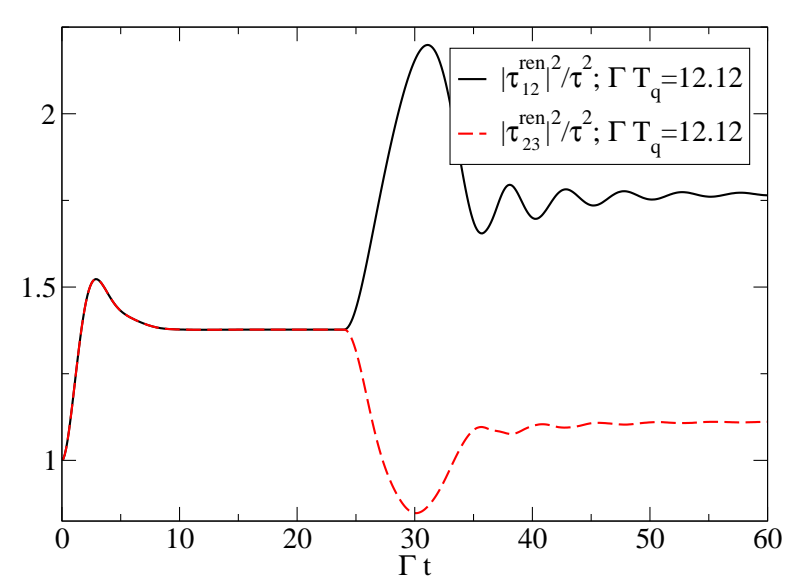

FIG. 5: (Color online) Renormalized hopping amplitudes across bonds $(1,2)$ and $(2,3)$ as a function of time (normalized to the bare time-independet value). The parameters are chosen as in Fig. 4 with $\Gamma T_{\mathrm{q}}=12.12$.

in Fig. 4. Interestingly, for $U>0$ and sufficiently large $T_{\mathrm{q}}$, $\bar{n}_{2}(t)$ increases for times slightly larger than $t_{\mathrm{q}}-T_{\mathrm{q}} / 2$ (see the dashed and dashed-dotted lines). For larger $T_{\mathrm{q}}$ the effect becomes more pronounced. There are two competing processes determining the occupancy $\bar{n}_{2}(t)$. (a) The increase of the onsite energy favors a depletion process and dominates in the case where the quench is abrupt. (b) At finite bias voltage the interaction induced renormalization of the hopping amplitudes across bonds $(1,2)$ and $(2,3)$ is not equal as shown in Fig. 5 . The hopping amplitude across bond $(1,2)$ (solid line), which is closer to the lead held at higher chemical potential, increases more than the hopping across $(2,3)$ (dashed line), by which charge is transported to the lead held at lower chemical potential. This induces a tendency towards an increase of the charge on the central site. When the increase of $\epsilon$ is performed slow enough the increase of $\bar{n}_{2}$ due to the hopping asymmetry can initially overcompensate the depletion. At larger times process (a) wins and the occupancy decreases exponentially (with overlayed oscillations). We emphasize that the (initial) increase results from the interplay of the finite bias voltage $V>0$, the two-particle interaction $U>0$, and the parameter change not being abrupt. It vanishes when either of these conditions is not fulfilled.

\section{The role of initial correlations}

In protocols (ii) and (iii) the two-particle interaction is turned on at $t=0$. Correlations are thus fully developed at the time of the abrupt or gradual increase of $\epsilon$. To investigate the role of these initial (with respect to $t_{\mathrm{q}}$ or $\left.T_{\mathrm{q}}-t_{\mathrm{q}} / 2\right)$ correlations in the quench dynamics we now modify the 'initially correlated' protocols (ii) and (iii) and abruptly turn on the interaction at $t_{\mathrm{q}}$ or $T_{\mathrm{q}}-t_{\mathrm{q}} / 2$, respectively. We will refer to these modified protocols as 


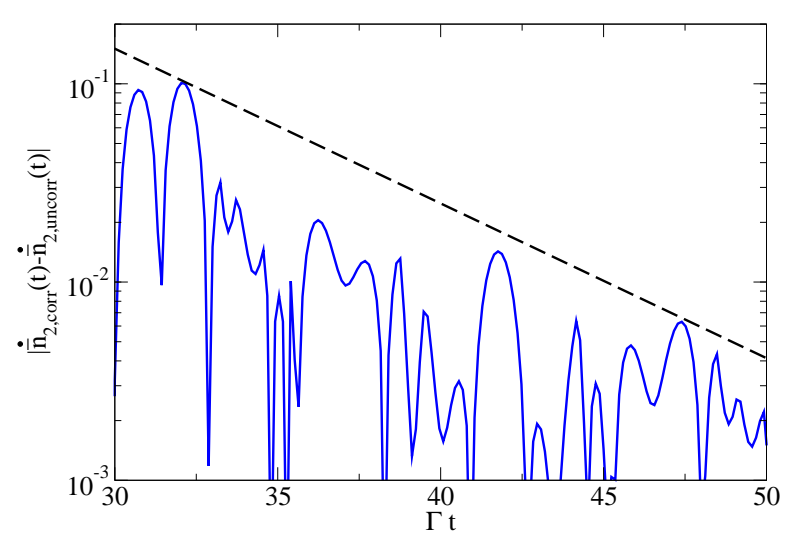

FIG. 6: (Color online) Absolute value of the difference of the derivatives of $\bar{n}_{2}(t)$ for the initially correlated and uncorrelated protocol (ii). The parameters are as in Fig. 2 The dashed line displays an exponential decay with the rate set by the final Hamiltonian. Note the linear-logarithmic scale.

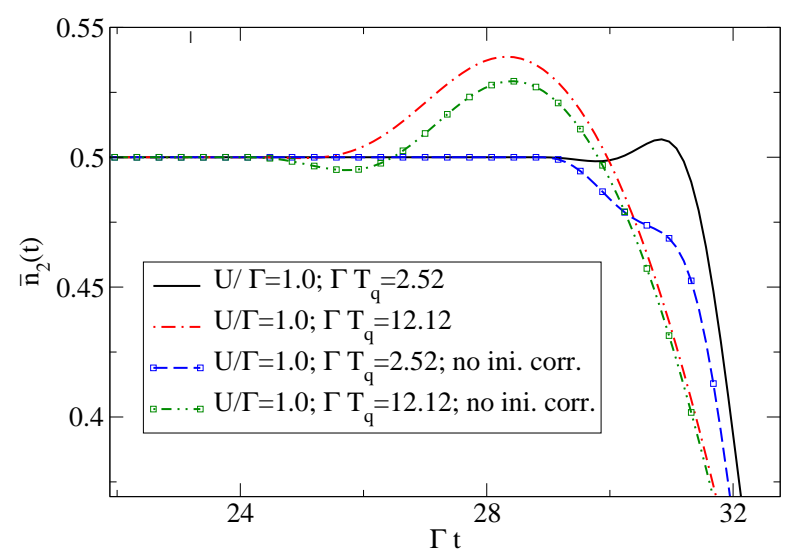

FIG. 7: (Color online) Comparison between the time evolution of $\bar{n}_{2}$ for protocol (iii) with and without initial correlations. A zoom-in of the interesting time regime in which $\epsilon$ is raised is shown.

'initially uncorrelated'.

We start out with the comparison of the time evolution of the initially correlated and uncorrelated protocol (ii). It is not surprising that for our tunnel coupled quantum dot setup the occupancies $\bar{n}_{2}(t)$ of the two situations become equal exponentially fast. In particular, the plateaulike features discussed in connection with Fig. 2 are also present in the initially uncorrelated setup. To make the differences clearly visible in Fig. [6 we show the absolute value of the derivative of the difference of the two occupancies $\bar{n}_{2}$ for $t>t_{\mathrm{q}}$ on a linear-logarithmic scale. The envelope shows a linear behavior which proofs that the correlations build up on an exponential scale. In fact, this scale is found to be the decay rate set by the final Hamiltonian (after the quench). An exponential decay with this rate is shown as the dashed line in Fig. 6.

In protocol (iii) we found that the correlation induced increase of $\bar{n}_{2}(t)$ sets in right after the time $t_{\mathrm{q}}-T_{\mathrm{q}} / 2$ at which $\epsilon$ starts to be gradually raised. It is thus rea- sonable to expect more obvious differences in the dynamics of the central sites occupancy for the initially correlated and uncorrelated states of this protocol. As Fig. 7 shows the local maximum described in subsection IIIC decreases in height if initial correlations are absent (dashed-dotted line with squares) up to the extend that the maximum disappears for the faster parameter change with $\Gamma T_{\mathrm{q}}=2.52$ (dashed line with squares). Additionally, the short time behavior right after the parameter change has been initiated changes significantly. In the slowest increase of $\epsilon$ with $\Gamma T_{\mathrm{q}}=12.12$ (dashed-dotted line with squares) first a minimum develops. The initial decrease of $\bar{n}_{2}$ reflects the crucial importance of two-particle correlations for the accumulation of charge on the central dot. After significant correlations have build up (in time) $\bar{n}_{2}(t)$ increases as found for the initially correlated protocol (iii). The long-time behavior of the initially correlated and uncorrelated protocols (iii) is indistinguishable.

\section{E. Protocol (iv)}

When studying time-dependent currents and level occupancies of correlated quantum dots in most theoretical studies the response towards turning on the level-lead couplings is considered. In experiments on the other hand one generically starts out of an equilibrium state in which the leads are coupled and at a certain time turns on the driving bias voltage. Next we therefore consider this experimentally motivated protocol (iv) in the scaling limit (the IRLM). For this we start out in the standard initial state with $V=0$ and empty dot following protocol (i). After a steady state is reached the bias voltage is quenched to a positive value. In Fig. $8 \bar{n}_{2}(t)$ for the abrupt voltage quench is compared to the evolution out of the standard initial state (with $V>0$ ) as discussed in subsection ЏA. The parameters are $V=10 T_{\mathrm{K}}$, $\tau / \Gamma=0.0025, \epsilon=10 T_{\mathrm{K}}$, and $U / \Gamma=0.2$ as well as 0 . The occupancy for the two cases shows sizable differences. While in the evolution out of the standard initial state the two frequencies $\left|\epsilon^{\mathrm{ren}} \pm V / 2\right|$ dominate (see subsection (IIA) the shape is deformed towards a (damped) triangular-like time dependence when the bias is only turned on at a later time; instead of two, many frequencies $\omega$ with $\epsilon^{\text {ren }}-V / 2<|\omega|<\epsilon^{\text {ren }}+V / 2$ contribute to this line shape. The deformation is already apparent for $U=0$. Furthermore, the amplitude of the oscillations is reduced when the bias voltage is quenched. In contrast to $\bar{n}_{2}(t)$, the line shapes of the current $J_{L}(t)$ in protocols (i) and (iv) shown in Fig. 9 are rather similar. The only prominent difference is the amplitude which decreases if the bias is turned on at a later time (also at $U=0$ ). The correlation effects of protocol (iv) for large $\epsilon$ are the renormalization of the decay rate $T_{\mathrm{K}}$ (note that the time axis of Figs. 8 and 9 is given in units of $T_{\mathrm{K}}$ ) and the slightly increased amplitude of the oscillation, both known from protocol (i).

In addition to the case of a large onsite energy we study 


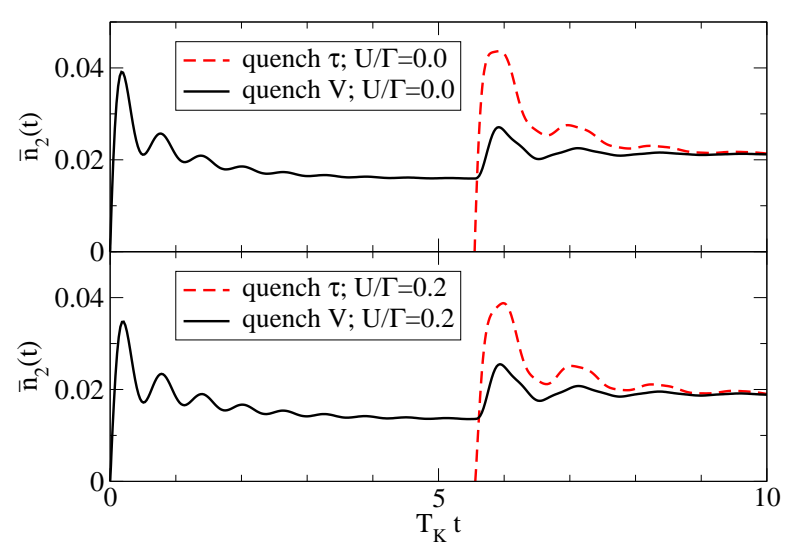

FIG. 8: (Color online) Time evolution of the occupancy $\bar{n}_{2}$ for a quench in the bias voltage (protocol (iv)) and the levellead coupling (protocol (i)). The parameters are $V=10 T_{\mathrm{K}}$, $\tau / \Gamma=0.0025, \epsilon=10 T_{\mathrm{K}}$, and $U / \Gamma=0.2$ as well as 0 . For a better comparison the data or protocol (i) are shifted along the time axis to the time of the voltage quench $T_{\mathrm{K}} t_{\mathrm{q}} \approx 5.5$.

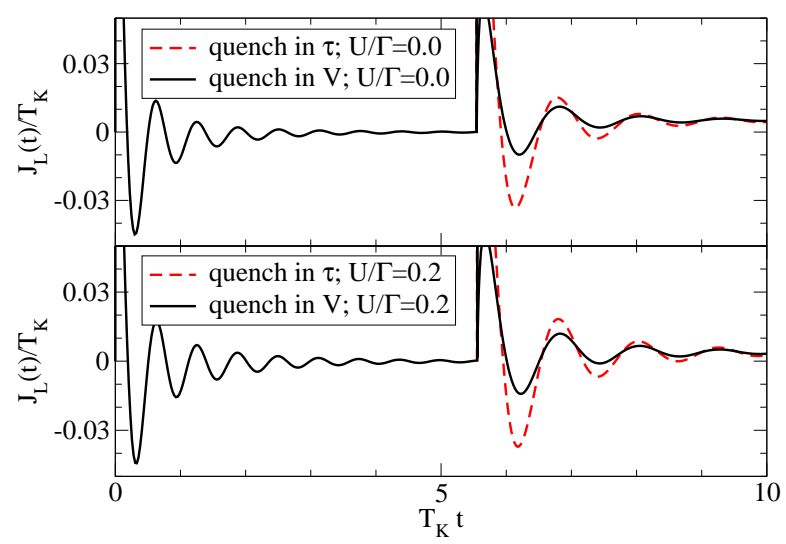

FIG. 9: (Color online) The same as in Fig. 8 but for the current $J_{L}(t)$.

the dynamics for $\epsilon=0$ and initial occupancy $\bar{n}_{2}(t=0)=0$. The steady-state occupancy equals $1 / 2$ and it is useful to consider the quantity $\left|2 \bar{n}_{2}(t)-1\right|$ on a logarithmic scale, which enhances the visibility of many features. For $V=0$ the IRLM is equivalent to the ohmic spin-boson mode ${ }^{28}$ and $\left|2 \bar{n}_{2}(t)-1\right|$ corresponds to the absolute value of the $z$-component of the spin expectation value. Figure 10 shows the time evolution of $\left|2 \bar{n}_{2}(t)-1\right|$ out of the standard initial state and a subsequent quench in the bias voltage from $V=0$ to $V=10 T_{\mathrm{K}}$ at $T_{\mathrm{K}} t_{\mathrm{q}}=10$. The noninteracting case as well as $U / \Gamma=0.1$ are depicted. The hopping is $\tau / \Gamma=0.0025$. For $U=0,\left|2 \bar{n}_{2}(t)-1\right|$ goes to zero purely exponentially with a single dominant rate $T_{\mathrm{K}}(U=0)$; straight line (dashed) on the linear-logarithmic scale of Fig. 10. This can be understood from the explicit analytical expression for $\bar{n}_{2}(t)$ in the noninteracting case e.g. given in Eq. (79) of Ref. 13. For $\epsilon=0$ the two oscillatory terms cancel. For $U>0$ one observes the exponentially damped oscillations with frequency $\sim U$ of the

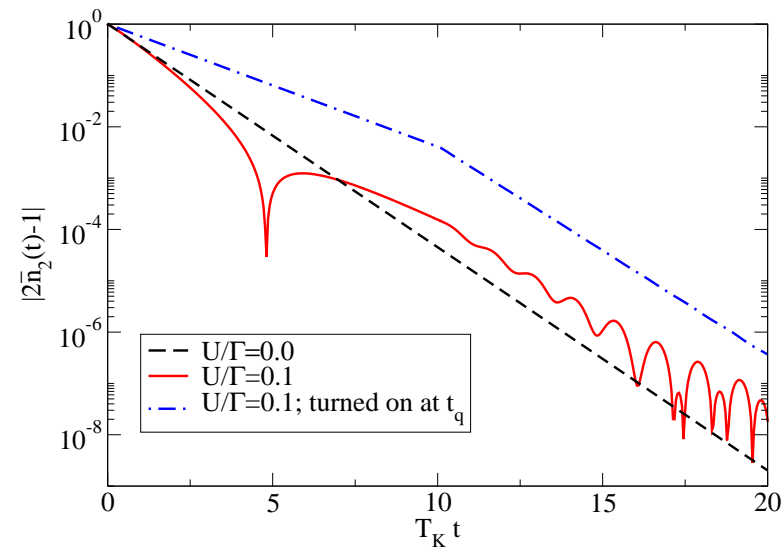

FIG. 10: (Color online) Time evolution of $\left|2 \bar{n}_{2}(t)-1\right|$ for a quench in the bias voltage from $V=0$ to $V=10 T_{\mathrm{K}}$ at $T_{\mathrm{K}} t_{\mathrm{q}}=10$. Results for $U / \Gamma=0.1$ as well as 0 are shown. The other parameters are $\tau / \Gamma=0.0025$ and $\epsilon=0.0$. For comparison a graph for a setup in which the interaction is turned on simultaneously with the bias is shown as well. Note the linear-logarithmic scale. The time axis is given in units of $T_{\mathrm{K}}(U=0)$ for the dashed line and $T_{\mathrm{K}}(U=0.1 \Gamma)$ for the solid and the dashed-dotted line. This leads to the two different slopes and the kink close to $t_{\mathrm{q}}$ in the dashed-dotted line.

coherent phase of the spin-boson model $13,29,30$ before the bias quench (dip for $t<t_{\mathrm{q}}$ of the solid line) and additional oscillations with much higher frequencies after this. Additionally, the dynamics of a setup in which $V$ as well as $U$ are turned on at $t_{\mathrm{q}}$ (no correlations for $t<t_{\mathrm{q}}$ ) is shown as the dashed-dotted line. Although the relaxation rate appears to change to its renormalized value almost instantly as the interaction is turned on (kink at $t \approx t_{\mathrm{q}}$ and the same slope as the other lines for $t>t_{\mathrm{q}}$; note that the time is given in units of the interacting $T_{\mathrm{K}}$ for all times), the dynamics differs significantly compared to the one of the solid line; on the scale of the plot no oscillations are visible. We conclude that in this example the buildup of the correlations quickly changes the relaxation rate but does not result in significant oscillations as found for the initially correlated case. We here concentrated on the occupancy, as the dynamics of $J_{L}$ proves less interesting and is characterized by an exponential approach of the steady-state value modulated by oscillations with frequency $V / 2$.

\section{SUMMARY}

We have studied the dynamics of the dot occupancy and the current resulting out of abrupt quenches and smooth parameter changes in the IRLM and variants of this. The time evolution shows characteristics which depend on the protocol with which the different parameters are varied in time. In particular, correlations can lead to a rich dynamics showing interesting effects which go beyond the renormalization of the decay rate found in the 
standard protocol (i). Using our approximate functional RG approach we were able to identify and explain several of those. Our method is very flexible, can be used for all time scales, and other time dependences of the model parameters than the ones considered here can be treated as well. We exemplified the role of initial correlations and determined the difference between the dynamics resulting out of switching-on the level-lead coupling in the presence of a finite bias voltage and the experimentally more relevant case of turning-on the bias voltage in an equilibrium state with coupled leads.

\section{Acknowledgments}

We thank S. Jakobs and C. Karrasch for collaborations in the initial state of this work as well as K. Schönhammer and $\mathrm{H}$. Schoeller for valuable discussions. This work was supported by the DFG via FOR 723.
1 G.D. Mahan, Many-Particle Physics (Plenum Press, New York, 1990).

${ }^{2}$ H. Bruus and K. Flensberg Many-Body Quantum Theory in Condensed Matter Physics (Oxford University Press, 2004).

3 R. Hanson, L.P. Kouwenhoven, J.R. Petta, S. Tarucha, and L.M.K. Vandersypen, Rev. Mod. Phys. 79, 1217 (2007).

4 A. Hackl, D. Roosen, S. Kehrein, and W. Hofestetter, Phys. Rev. Lett. 102, 196601 (2009).

5 S. Kehrein, The Flow Equation Approach to Many-Particle Systems (Springer Verlag, Berlin, 2010).

6 M. Pletyukhov, D. Schuricht, and H. Schoeller, Phys. Rev. Lett. 104, 106801 (2010).

7 H. Schoeller and J. König, Phys. Rev. Lett. 84, 3686 (2000); H. Schoeller, Eur. Phys. J. Spec. Top. 168, 179 (2009).

8 F.B. Anders and A. Schiller, Phys. Rev. B 74, 245113 (2006).

9 P. Schmitteckert, Phys. Rev. B 70, 121302(R) (2004).

10 F. Heidrich-Meisner, A.E. Feiguin, and E. Dagotto, Phys. Rev. B 79235336 (2009).

11 S. Weiss, J. Eckel, M. Thorwart, and R. Egger, Phys. Rev. B 77, 195316 (2008).

12 T.L. Schmidt, P. Werner, L. Mühlenbacher, and A. Komnik, Phys. Rev. B 78, 235110 (2008).

13 D. Kennes, S.G. Jakobs, C. Karrasch, and V. Meden, Phys. Rev. B 85, 085113 (2012).

14 W. Metzner, M. Salmhofer, C. Honerkamp, V. Meden, and K. Schönhammer, Rev. Mod. Phys. 84, 299 (2012).

15 S.G. Jakobs, V. Meden, and H. Schoeller, Phys. Rev. Lett. 99, 150603 (2007).

16 R. Gezzi, Th. Pruschke, and V. Meden, Phys. Rev. B 75,
045324 (2007).

17 S.G. Jakobs, Ph.D. thesis, RWTH Aachen University (2010), http://d-nb.info/1009075535.

18 C. Karrasch, M. Pletyukhov, L. Borda, and V. Meden, Phys. Rev. B 81, 125122 (2010).

19 C. Karrasch, Ph.D. thesis, RWTH Aachen University (2010), http://d-nb.info/100962234X, arXiv:1009.3852

20 C. Karrasch, T. Enss, and V. Meden, Phys. Rev. B 73, 235337 (2006).

21 C. Karrasch, R. Hedden, R. Peters, Th. Pruschke, K. Schönhammer, and V. Meden, J. Phys.: Condens. Matter 20, 345205 (2008).

22 S.G. Jakobs, M. Pletyukhov, and H. Schoeller, Phys. Rev. B 81, 195109 (2010).

23 P. Schlottmann, Phys. Rev. B 22, 613 (1980); ibid. 25, 4815 (1982).

24 D. Bohr and P. Schmitteckert, Phys. Rev. B 75, 241103 (2007)

25 L. Borda, A. Schiller, and A. Zawadowski, Phys. Rev. B 78, 201301 (2008).

26 S. Andergassen, M. Pletyukhov, D. Schuricht, H. Schoeller, and L. Borda, Phys. Rev. B 83, 205103 (2011); ibid. S. Andergassen, M. Pletyukhov, D. Schuricht, H. Schoeller, and L. Borda, Phys. Rev. B 84, 039905(E) (2011).

27 G. Stefanucci and C.-O. Almbladh, Phys. Rev. B 69, 195318 (2004).

28 A.J. Legett, S. Chakravarty, A.T. Dorsey, M.P.A. Fisher, A. Garg, and W. Zwerger, Rev. Mod. Phys. 59, 1 (1987).

29 R. Egger, H. Grabert, and U. Weiss, Phys. Rev. E 55, R3809 (1997).

${ }^{30}$ F. Lesage and H. Saleur, Phys. Rev. Lett. 80, 4370 (1998). 